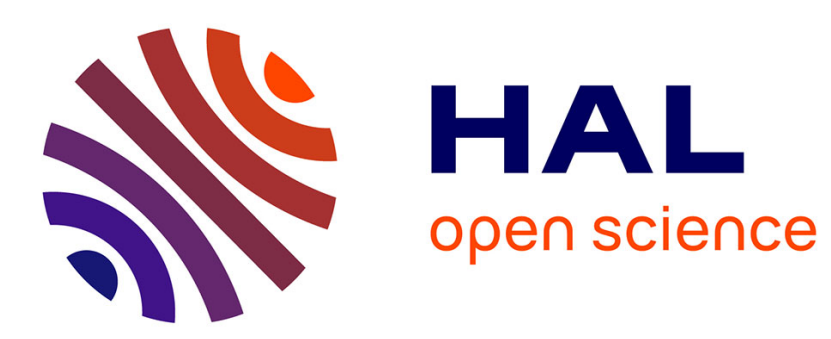

\title{
RANS modelling of a high-temperature jet in a cold crossflow: from eddy viscosity models to advanced anisotropic approaches
}

\author{
Romain Paysant, Emmanuel Laroche, Pierre Millan, Pierre Buet
}

\section{To cite this version:}

Romain Paysant, Emmanuel Laroche, Pierre Millan, Pierre Buet. RANS modelling of a hightemperature jet in a cold crossflow: from eddy viscosity models to advanced anisotropic approaches. AIAA Scitech 2021 Forum, Jan 2021, Virtual event, United States. pp.AIAA 2021-1542, 10.2514/6.2021-1542 . hal-03414431

\section{HAL Id: hal-03414431 \\ https://hal.science/hal-03414431}

Submitted on 4 Nov 2021

HAL is a multi-disciplinary open access archive for the deposit and dissemination of scientific research documents, whether they are published or not. The documents may come from teaching and research institutions in France or abroad, or from public or private research centers.
L'archive ouverte pluridisciplinaire HAL, est destinée au dépôt et à la diffusion de documents scientifiques de niveau recherche, publiés ou non, émanant des établissements d'enseignement et de recherche français ou étrangers, des laboratoires publics ou privés. 


\title{
RANS modelling of a high-temperature jet in a cold crossflow: from eddy viscosity models to advanced anisotropic approaches
}

\author{
Romain Paysant* \\ Airbus Helicopters S.A.S., 13725 Marignane, France \\ Emmanuel Laroche ${ }^{\dagger}$ \\ ONERA / DMPE, Université de Toulouse, F-31055 Toulouse, France \\ Pierre Millan \\ ONERA / DMPE, Université de Toulouse, F-31055 Toulouse, France \\ Pierre Buet ${ }^{\S}$ \\ Airbus Helicopters S.A.S., 13725 Marignane, France
}

The present work investigates the ability of several Reynolds Averaged Navier-Stokes (RANS) turbulence models to predict flow dynamics and mixing of a high-temperature turbulent jet in a cold crossflow. Two turbulence models relying on an isotropic eddy viscosity assumption were tested. They globally capture the main flow features but strongly underestimate turbulent mixing. The Explicit Algebraic Reynolds Stress Model (EARSM) does not bring significant improvement over isotropic RANS models on this configuration. Reynolds Stress Models (RSM) predict a more diffuse jet and are in better agreement with the measurements. Influence of turbulent heat flux modelling is also assessed for RSM and EARSM models. For this purpose, the Generalized Gradient Diffusion Hypothesis (GGDH) is used instead of the classical Gradient Diffusion Hypothesis (GDH). This advanced thermal modelling enhances thermal field prediction creating extra diffusion compared to the latter which involves a constant turbulent Prandtl number.

\section{Nomenclature}

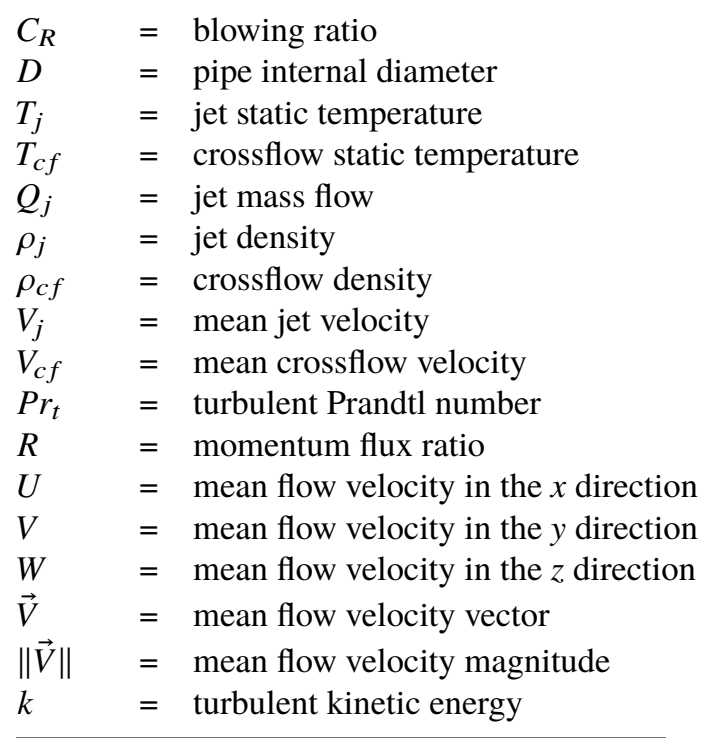

*PhD student, Thermal Engineering Department.

${ }^{\dagger}$ Research Scientist, MultiPhysics and Energetics Department.

†Director of MultiPhysics and Energetics Department, MultiPhysics and Energetics Department.

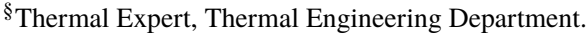


$\omega \quad=\quad$ specific dissipation rate

$\varepsilon=$ turbulent dissipation

$v_{t} \quad=$ turbulent (or eddy) viscosity

$\left\langle u_{i}^{\prime} u_{j}^{\prime}\right\rangle=$ Reynolds stress tensor

$\left\langle u_{i}^{\prime} T^{\prime}\right\rangle=$ turbulent heat flux vector

\section{Introduction}

Jets in crossflows are frequently encountered in either natural or industrial systems. Among the extensive list of jet in crossflow applications, one can cite film cooling for turbines and combustors, fuel injection and dilution jets in combustors, pollutants and plumes dispersion from smokestacks or volcanoes, lifting jets of Vertical and/or Short Take-Off and Landing (V/STOL) aircrafts. In the present paper, a jet in crossflow configuration representative of the ejection of helicopter engine exhaust gases is simulated. The distinctive feature of this configuration is the strong temperature difference between jet and crossflow as exhaust gases are ejected at a temperature of about $600^{\circ} \mathrm{C}$ to $700^{\circ} \mathrm{C}$. Interaction of the hot gases plume with rotor flow and relative wind can lead to two kinds of thermal issues: thermal aggression of helicopter external parts and hot gases reingestion through engines air intakes and avionic bay ventilations. Thus, being able to assess numerically exhaust gases trajectory and mixing is of crucial interest for design purpose.

Experimental works dealing with high-temperature jets in cold crossflows are scarce. Prior studies have mainly focused on ambient temperature or slightly heated jets in order to measure wall heat flux. Kamotani \& Greber [1] were among the first to investigate configurations with large temperature difference between jet and crossflow. They studied the influence of jet to crossflow temperature difference, $\Delta T$, for two momentum flux ratios $R=\rho_{j} V_{j}^{2} / \rho_{c f} V_{c f}^{2}=59.6$ and 15.3 where $\rho_{j}, V_{j}, \rho_{c f}$ and $V_{c f}$ are respectively the density and mean velocity of jet and crossflow. The authors considered two jet temperatures corresponding to $\Delta T=T_{j}-T_{c f}=24^{\circ} \mathrm{C}$ and $160^{\circ} \mathrm{C}$. At moderate $R$, they observed that lines of local temperature maxima were coincident for both $\Delta T$. However, at higher $R$, this line gets closer to the wall as $\Delta T$ increases, highlighting a dependence on jet to crossflow density ratio. Harms [2] reports a greater spreading of the counter-rotating vortices in the case of a hot jet compared to a cold one at the same momentum flux ratio $(R=64)$.

From a numerical point of view, several studies were dedicated to RANS modelling of jet in crossflow but mostly focused on flow dynamics. One can cite the work of Chochua et al. [3], Karvinen \& Ahlstedt [4], Ray et al. [5] and Li et al. [6]. Most of these studies agree that RANS models are qualitatively able to capture main flow characteristics such as jet trajectory, contrarotative vortex pair or recirculation zones but fail to predict correct levels of turbulent kinetic energy. On the contrary, few studies investigated turbulent mixing. Bézard et al. [7] studied turbulent mixing of a jet at moderate temperature $\left(T_{j}=80^{\circ} \mathrm{C}\right)$ and low momentum flux ratio $(R=0.64)$ using $k-\omega$ SST [8]. They showed that the lateral spreading of film-cooling effectiveness profiles at the wall is underestimated although velocity field is reasonably well predicted. On such low momentum flux ratio configurations, Lakehal [9] and Bergeles et al. [10] demonstrated enhanced thermal field prediction with the use of a near-wall anisotropic eddy viscosity model obtained by modifying eddy viscosity in transverse directions. Results were further improved using a near-wall model for the turbulent Prandtl number. However, their studies were restricted to low injection rates configurations in which jet remains attached to the wall. A jet at higher temperature $\left(T_{j} \approx 500^{\circ} \mathrm{C}\right)$ and momentum flux ratio $(R=5.41)$ was studied by Rusch et al. [11]. They investigated the influence of turbulence models for fire simulations. However, velocities involved were very low $\left(V_{j}=2.71 \mathrm{~m} . \mathrm{s}^{-1}\right.$ and $\left.V_{c f}=0.73 \mathrm{~m} \cdot \mathrm{s}^{-1}\right)$ and jet developed in a confined environment.

\section{Experiments}

As mentioned above, experimental data for hot jets in crossflows are scarce. Therefore, experiments were conducted in ONERA's F2 large research-type low speed wind tunnel in order to build up a detailed database to validate simulations. Tested configuration (Fig. 11) consisted of a pipe of internal diameter $D=40 \mathrm{~mm}$ flush-mounted on a flat plate. A fin is placed $10 D$ downstream jet ejection to assess hot jet interaction with structure.

Jet mass flow and temperature were set to $Q_{j}=70 \mathrm{~g} . \mathrm{s}^{-1}$ and $T_{j}=400^{\circ} \mathrm{C}$ leading to a mean jet velocity $V_{j}=106.2 \mathrm{~m} . \mathrm{s}^{-1}$ and a jet density $\rho_{j}=0.52 \mathrm{~kg} \cdot \mathrm{m}^{-3}$. Crossflow freestream velocity was set to $V_{c f}=30 \mathrm{~m} \cdot \mathrm{s}^{-1}$ and its temperature was maintained to $T_{c f}=20^{\circ} \mathrm{C}$. A turbulent boundary layer develops on the flat plate upstream jet ejection. Laser Doppler Velocimetry (LDV) measurements performed $4 D$ upstream jet ejection show a boundary layer thickness $\delta_{99 \%}$ ranging from 3 to $4 \mathrm{~mm}$ depending on spanwise location. At pipe outlet, the jet exhibits a fully developed velocity profile whose symmetry has been checked without crossflow. The blowing ratio $C_{R}$ is 1.54, the momentum flux ratio $R$ is 5.45. Particle Image Velocimetry (PIV), Background Oriented Schlieren (BOS), thermocouple and LDV measurements were 


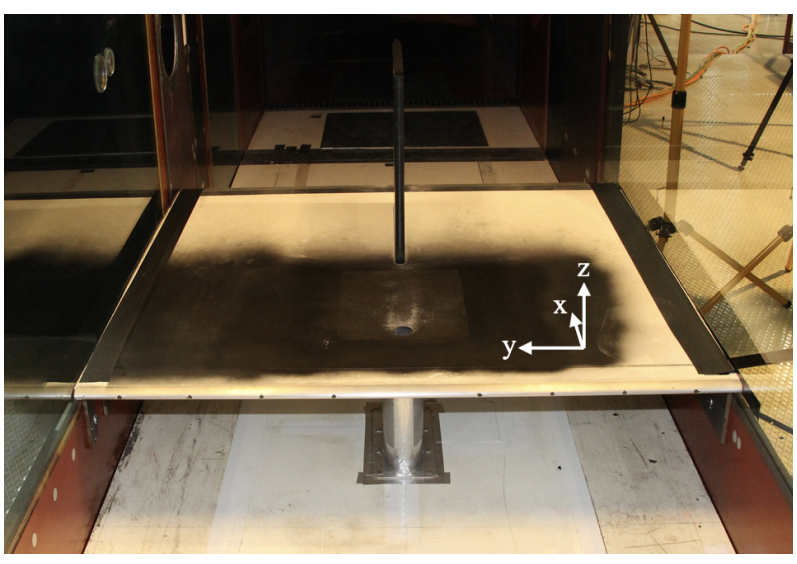

Fig. 1 View of the tested jet in crossflow configuration

performed. In addition, infrared thermography measurements were carried out over fin surface. For further information concerning these experiments, the reader will be referred to an ongoing dedicated paper.

\section{Numerical Simulations}

Reynolds Averaged Navier-Stokes simulations were performed on the configuration presented above with ONERA's finite-volume unstructured compressible code CEDRE. Fluxes were computed using a HLLC scheme coupled to a Van Leer flux limiter. Green's method was used for gradient computation. A $1^{\text {st }}$ order Euler implicit scheme was selected for temporal integration. The system was solved with an iterative GMRES method.

A structured mesh composed of 1.98 millions hexahedral elements was generated for the calculations. Over pipe and flat plate walls, first cell height was set to $10^{-3} \mathrm{~m}$ leading to $\mathrm{y}^{+} \approx 50$ everywhere in the domain except in recirculation areas located upstream and downstream jet ejection where low velocities tend to decrease $\mathrm{y}^{+}$value. Thus, wall functions were used for all calculations. This choice arises from the observation that solutions got stuck in a dissymmetrical state when solving flow down to the viscous sublayer $\left(\mathrm{y}^{+} \leq 1\right)$. This behaviour could not be explained by mesh symmetry or solver settings issues. However, the use of wall functions is not detrimental for the present work as flat plate heat flux was of minor interest due to the relatively important jet lift-off.

Numerical boundary conditions were chosen to match experimental ones. The wind tunnel and pipe inlets of the computational domain, both placed $4 D$ upstream jet ejection location, are modelled using a mass flux inlet condition. For wind tunnel inlet, a uniform temperature distribution of $T_{c f}=20^{\circ} \mathrm{C}$ and a mass flux profile were prescribed based on LDV measurements. Profiles of mass flux, turbulent quantities and total temperature were prescribed at pipe inlet using a preliminary RANS calculation of a periodic pipe. Pipe inlet conditions were validated afterwards by comparison between LDV measurements and simulated solution at pipe outlet. Concerning computational domain walls, only the flat plate and pipe were modelled using a no-slip condition while a slipping condition has been applied to sides and top walls. All walls are modelled with an adiabatic condition. Finally, the computational domain outflow boundary is modelled using a pressure outlet condition with a constant value equal to an ambient pressure of $1013.25 \mathrm{hPa}$.

Convergence has been carefully verified for each calculation. In addition to residuals decrease, probes have been created to monitor several variables evolution and their associated mean values. Mass flow imbalance between inlets and outlets was also checked to be lower than $1 \%$ of the smallest injected mass flow when a converged state is reached.

\section{A. First order closure turbulence models}

First calculations were carried out with two isotropic eddy viscosity models (EVM): Dutoya's $k-\varepsilon$ and Menter's $k-\omega$ SST [8] and with the anisotropic Explicit Algebraic Reynolds Stress Model (EARSM) proposed by Hellsten [12].

\section{Flow dynamics}

Profiles of vertical velocity $W$ in the centreplane $Y=0$ are presented on Fig. 2 It can be seen that $W$ profiles in the centreplane exhibit two distinct peaks. The first peak or upper peak, which is present since the beginning of jet development is due to the combination of the vertical component of jet velocity and crossflow deflection by the jet. The 

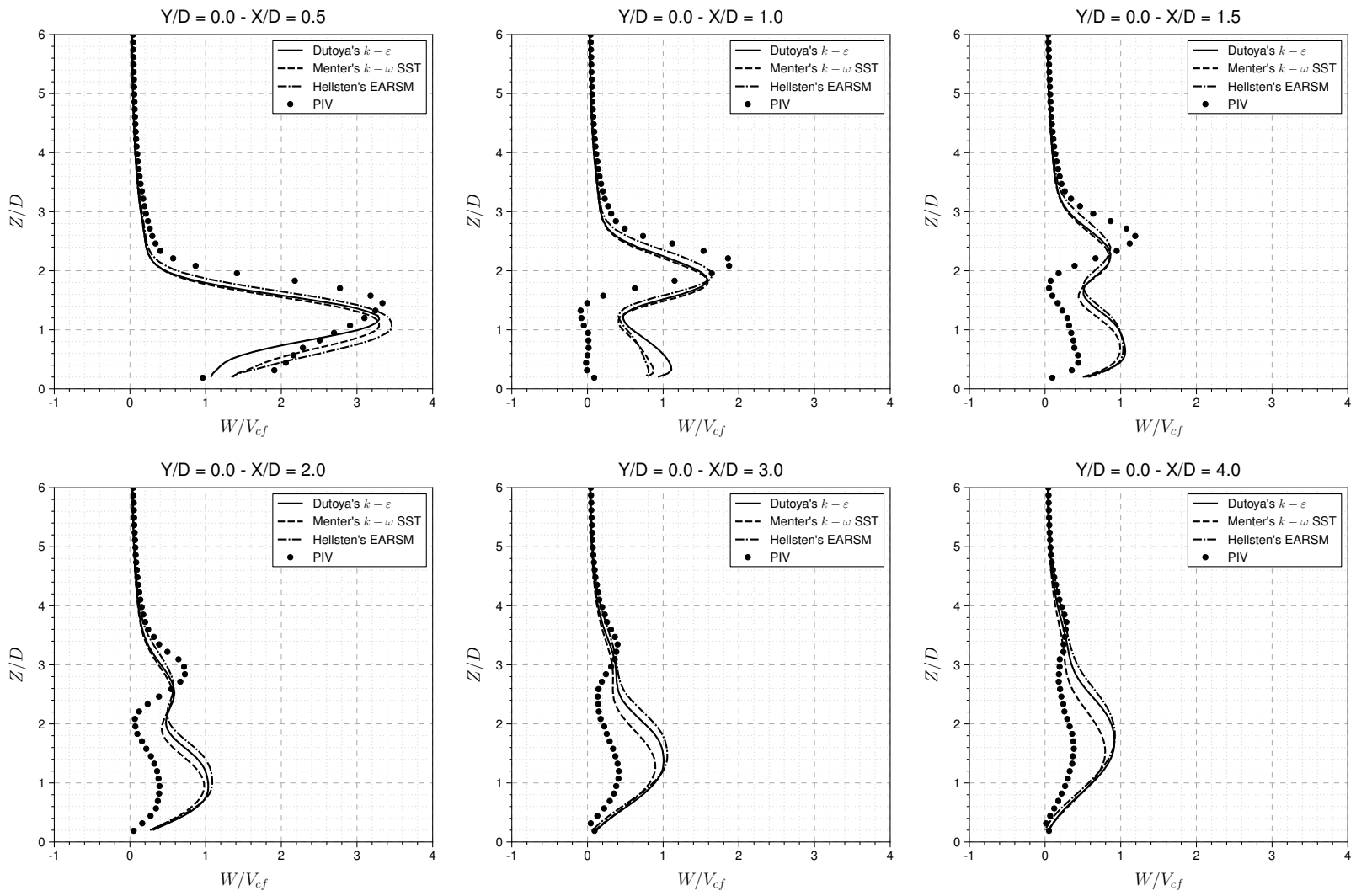

Fig. 2 Profiles of vertical velocity $W$ normalized by crossflow velocity $V_{c f}$ in the centreplane $Y=0$. Simulations performed with first order closure turbulence models.

intensity of this peak decreases with the distance downstream as the jet gets progressively aligned with the crossflow. Along jet development, one can also notice the progressive appearance of a secondary peak located closer to the wall. This secondary peak indicates the establishment of the counter-rotating vortex pair which induces an upward motion of the flow in the centreplane. Profiles simulated using EVM or EARSM turbulence models show that the intensity of the upper peak is globally well predicted at $X / D=0.5$ although it is slightly underestimated. On the contrary, its altitude is underestimated highlighting that jet bending over occurs too early in the simulations. The slight underestimation of the upper peak is also caused by the premature bending of the jet by the crossflow. This premature bending leads to a hasty reorientation of a larger amount of jet momentum, initially oriented along $Z$, along the crossflow direction. As a consequence, one can notice that the peak observed in the corresponding $U$ profile is slighlty overestimated at the same location (see Fig. 3). As $X / D$ increases, the intensity of the upper peak of $W$ decreases too fast in the simulations and its altitude remains too low even if it tends to catch-up with measurements. However, the most striking difference between measured and simulated $W$ profiles lies in the overestimation and premature appearance of the secondary peak. This observation indicates an earlier establishment as well as an overpredicted intensity of counter-rotating vortices by all turbulence models. Hellsten's EARSM model [12] which is designed to better capture turbulence anisotropy does not bring major improvement over models relying on an isotropic eddy viscosity hypothesis.

Profiles of the longitudinal velocity $U$, presented in Fig. 3, are slightly more complex than $W$ ones. In fact, they exhibit several distinct regions highlighting different physical characteristics of a jet in crossflow. For the highest $Z / D$ values, a freestream region where crossflow is unaffected by the presence of the jet $\left(U / V_{c f}=1\right)$ can be seen. As $Z / D$ decreases, one can notice a small drop in $U$ velocity which is only seen at $X / D=0.5$ and 1 . It is caused by the blockage effect induced by the jet upon the cross-stream. This small decrease is followed by a strong longitudinal velocity gradient typical of a shear-layer region which materialises the outer edge of the jet. As $Z / D$ continues to decrease, one can see the jet core region. It is characterised by a strong velocity overshoot (up to 2.1 times $V_{c f}$ from measurements) which progressively vanishes as the distance downstream $X / D$ increases. Below jet core region, a second shear-layer region with a fairly strong longitudinal velocity gradient can be observed. It connects the jet core to the wake region. 

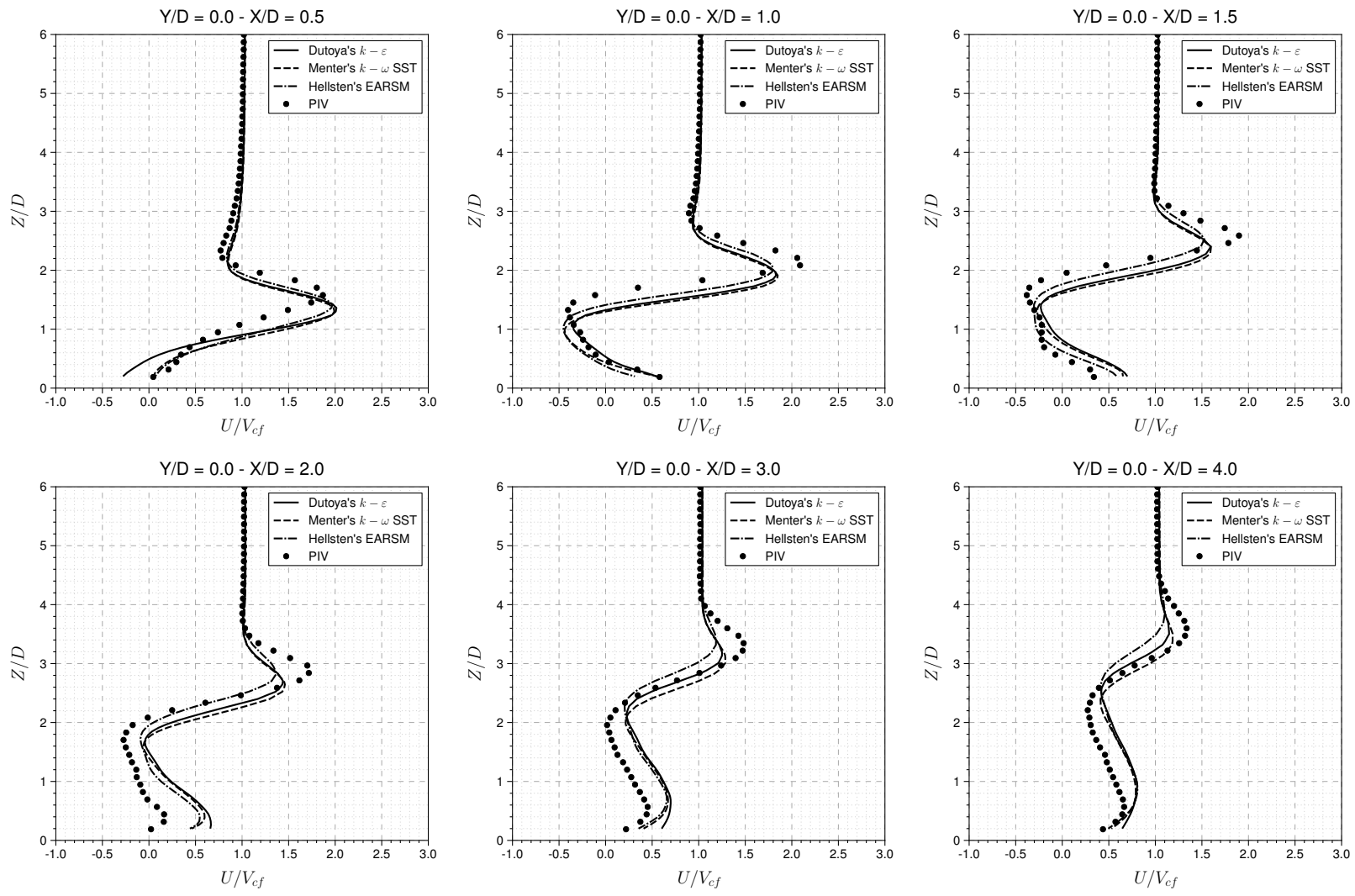

Fig. 3 Profiles of longitudinal velocity $U$ normalized by crossflow velocity $V_{c f}$ in the centreplane $Y=0$. Simulations performed with first order closure turbulence models.

In fact, $U$ profiles clearly show a wake behaviour of the flow in the lee of the jet characterised by a strong velocity deficit. This behaviour is even more pronounced as $X / D$ is low. The velocity deficit in the wake region is gradually recovered as $X / D$ increases. Finally, one can notice a small acceleration in the $U$ profile in the vicinity of the wall which gradually appears as $X / D$ increases. Fig. 3 shows that EVM and EARSM turbulence models qualitatively capture all characteristics of the jet mentioned above. At $X / D=0.5$, it can be seen that the velocity overshoot is overestimated and its altitude is too low as previously noticed for $W$ profile. Same conclusions applies here saying jet premature bending over reduces initial jet lift-off and reorients a greater part of jet initial momentum along cross-stream direction. As $X / D$ increases, the velocity overshoot decay is, at first, significantly faster than what is experimentally observed and its altitude remains too low. However, further downstream, this decrease slows down and altitude increases faster (even faster for EARSM) so simulated results catch-up with experimental ones. Results also show that the wake behaviour of the flow and recirculation zone located in the lee-side of the jet are relatively well reproduced (especially up to $X / D=1.5$ ). From $X / D=2$, it can be seen that simulated recovery of $U$ deficit in the wake is slightly overestimated. This is most likely due to the increased convection of high-momentum fluid towards the wall by the counter-rotating vortices induced motion which is overestimated.

Fig. 4 presents jet trajectory and decay of normalized velocity magnitude $\|\vec{V}\| / V_{j_{\max }}$ along jet trajectory where $V_{j_{\max }}$ is the jet exit velocity at pipe center. In the present study, jet trajectory is based upon local $U$ maxima in the centreplane. Velocity profiles in the centreplane were presented earlier up to $X / D=4$ as they flatten out beyond this location and no additional information comes out. Nevertheless, measurements were carried out up to $X / D=8$. Trajectory plots offer a clearer view of jet lift-off evolution over a longer distance downstream (up to $X / D=8$ ). One can see from Fig. 4 that both isotropic EVM provide a realistic forecast of jet trajectory. In contrast, the trajectory obtained with the anisotropic EARSM model is in good agreement with measurements up to $X / D=3$ but diverges further downstream. On the other hand, velocity magnitude decay along trajectory is an indicator of mixing of high-momentum fluid initially located within jet core. As mentioned before, it can be seen that jet lift-off is initially underestimated by all first order closure turbulence models. In addition, one can see that EVM and EARSM largely overestimate the initial decay of $\|\vec{V}\|$ 

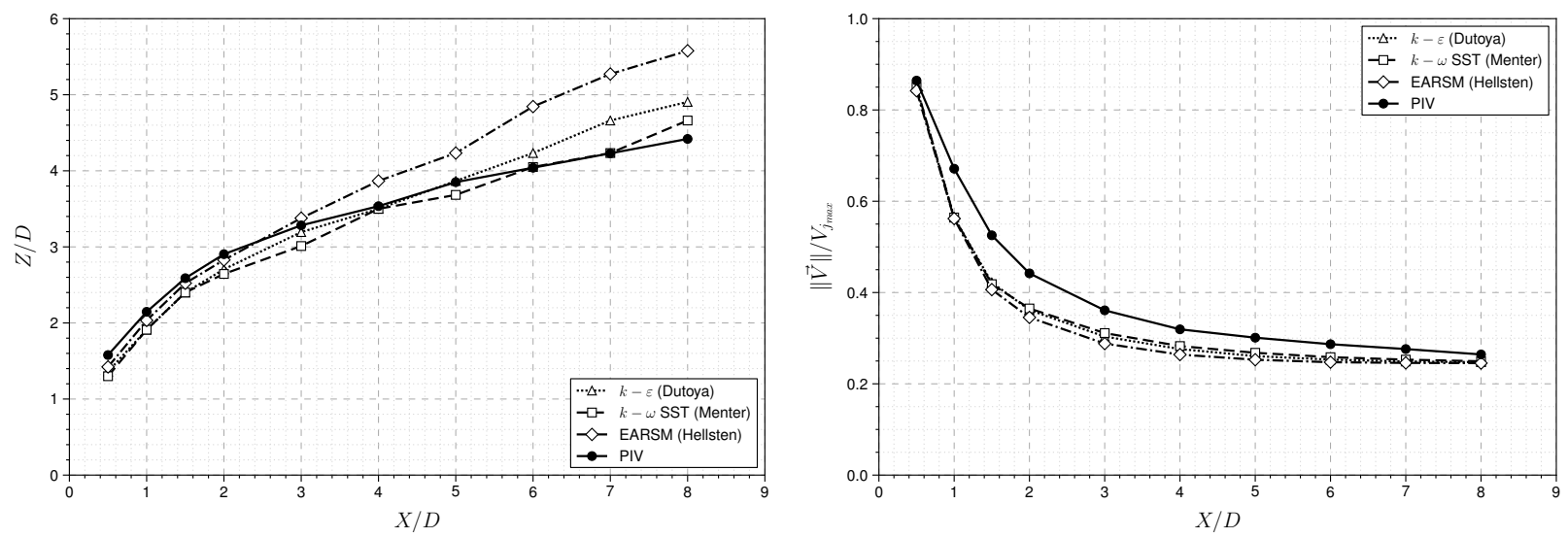

Fig. 4 Jet trajectory based on $U$ maxima in the centreplane (left) and decay of normalized velocity magnitude along jet trajectory (right).

predicting a steeper gradient and a rapid decrease of velocity magnitude. Further downstream, $\|\vec{V}\|$ decay slows down as $\|\vec{V}\|$ reaches its asymptotic value $V_{c f}$. In addition, the trajectory plots reveal that, beyond $X / D=5$, predicted trajectory slopes are too steep. From $X / D=5$, trajectories slopes differ by a factor of about 1.6 for the $k-\omega$ SST, 1.8 for the $k-\varepsilon$ and up to 2.2 for the EARSM with respect to the experimental one. Therefore, from $X / D=5$, the trend is reversed and simulations now underestimate jet deflection and lift-off increases much faster than experimentally observed. Haven \& Kurosaka [13, 14] have pointed out that jet lift-off is closely related to counter-rotating vortices strength, the latter promoting jet lift-off by counteracting crossflow bending effect. Comparison of $W$ profiles (Fig. 2) with trajectory and $\|\vec{V}\|$ decay plots (Fig. 4 ) confirms the strong correlation between counter-rotating vortices strength, jet lift-off and $\|\vec{V}\|$ decay. Indeed, the larger the overestimation of the counter-rotating vortices intensity, the higher the jet lift-off and the faster the decay in velocity magnitude. It is assumed that the earlier establishment of the counter-rotating vortices as well as the overestimation of their intensity in the calculations result in an increased convection of high-momentum fluid from jet core towards other jet areas. Nevertheless, a fair prediction of counter-rotating vortex pair intensity is not only essential for proper prediction of flow dynamics but is also of first order for correct thermal field prediction.

\section{Thermal aspects}

Simulated temperature contours in several transverse planes $(X / D=0.5,1.5$ and 3) were plotted for each calculation and compared to experimental measurements (see Fig. 5). Results show that both EVM underestimate turbulent mixing leading to overestimated temperature levels within jet plume. As an example, in the transverse plane located at $X / D=3$, maximum temperature is overestimated by $+32^{\circ} \mathrm{C}$ for the $k-\omega \mathrm{SST}$ and $+47.5^{\circ} \mathrm{C}$ for the $k-\varepsilon$ with respect to thermocouples measurements (maximum measured temperature at $X / D=3$ is $153^{\circ} \mathrm{C}$ ). In addition, topology of simulated thermal fields strongly differs from the measured one. On the one hand, measurements show that temperature maxima remains located within jet core up to $X / D=3$. On the other hand, calculations evidence a rapid migration of temperature maxima from jet core to vortices cores. Kamotani \& Greber [1] demonstrated that temperature maxima could either be located in jet core or migrate towards vortex centers depending on counter-rotating vortices strength. Results presented here are in agreement with their observations. The overestimation of counter-rotating vortices strength by EVM causes the rapid migration of temperature maxima towards vortices cores. Finally, EVM simulations also exhibit a compact jet plume with strong temperature gradients along jet envelope as can be seen on Fig. 5. In contrast, experiments (first row of Fig. 5) evidence a much more diffused jet plume with smoother temperature gradients. The use of the anisotropic EARSM model proposed by Hellsten [12] brings a slight improvement regarding thermal field prediction. Jet plume diffusion is slightly increased over results given by both EVM but is still underestimated compared to experimental measurements. In the transverse plane located at $X / D=3$, maximum temperature is overestimated by $+27^{\circ} \mathrm{C}$ compared to measured one which is slightly lower than EVM predictions. However, thermal field prediction by the EARSM suffers from its inability to properly capture counter-rotating vortices intensity. This drawback is clearly visible on temperature contours plot in the transverse plane located at $X / D=3$ where temperature maxima are located inside vortices cores which strongly differs from experimental findings. 

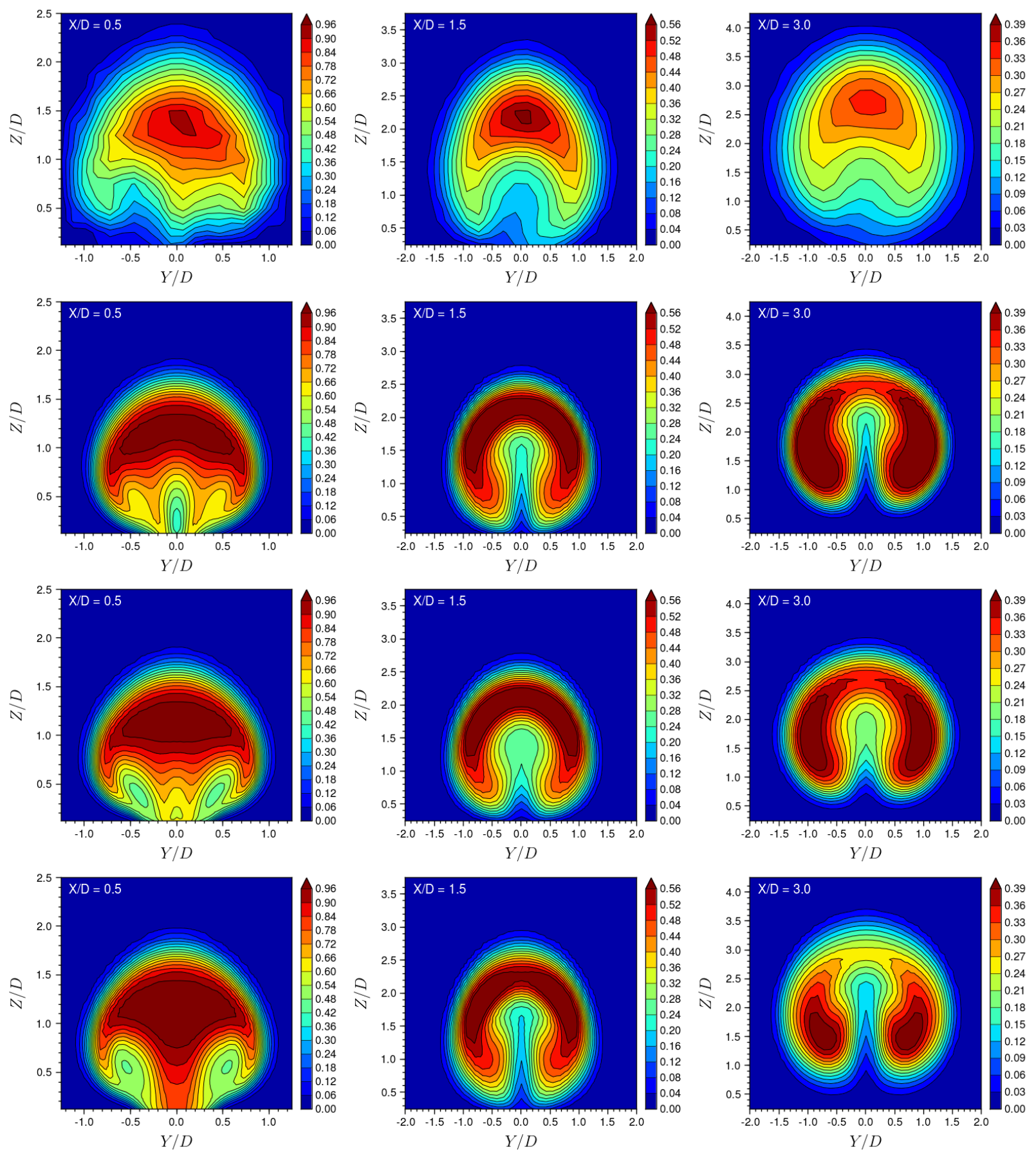

Fig. 5 Normalized temperature fields $\left(T-T_{c f}\right) /\left(T_{j}-T_{c f}\right)$ in three transverse planes downstream jet ejection. Measurements ( $\left(^{\text {st }}\right.$ row $)$, Dutoya's $k-\varepsilon\left(2^{\text {nd }}\right.$ row $)$, Menter's $k-\omega$ SST [8] $\left(3^{\text {rd }}\right.$ row $)$ and Hellsten's EARSM [12] (4h row).

\section{B. Elliptic Blending Reynolds Stress Models (EBRSM)}

Calculations with higher-order closure turbulence models were also carried out. Compared to EVM $\left(\left\langle u_{i}^{\prime} u_{j}^{\prime}\right\rangle\right.$ computed with the help of an eddy viscosity hypothesis) or EARSM $\left(\left\langle u_{i}^{\prime} u_{j}^{\prime}\right\rangle\right.$ computed with the help of an algebraic equation) these models solve a transport equation for each component of the Reynolds stress tensor. Therefore, these approaches are more prone to deal with complex flows as they rely on less restrictive hypotheses and a lower degree of empiricism. In the present study, two Elliptic Blending Reynolds Stress Models (EBRSM) were used: an $\omega$-based 
model (Aupoix [15]) and an $\varepsilon$-based model (Manceau [16]).

\section{Flow dynamics}
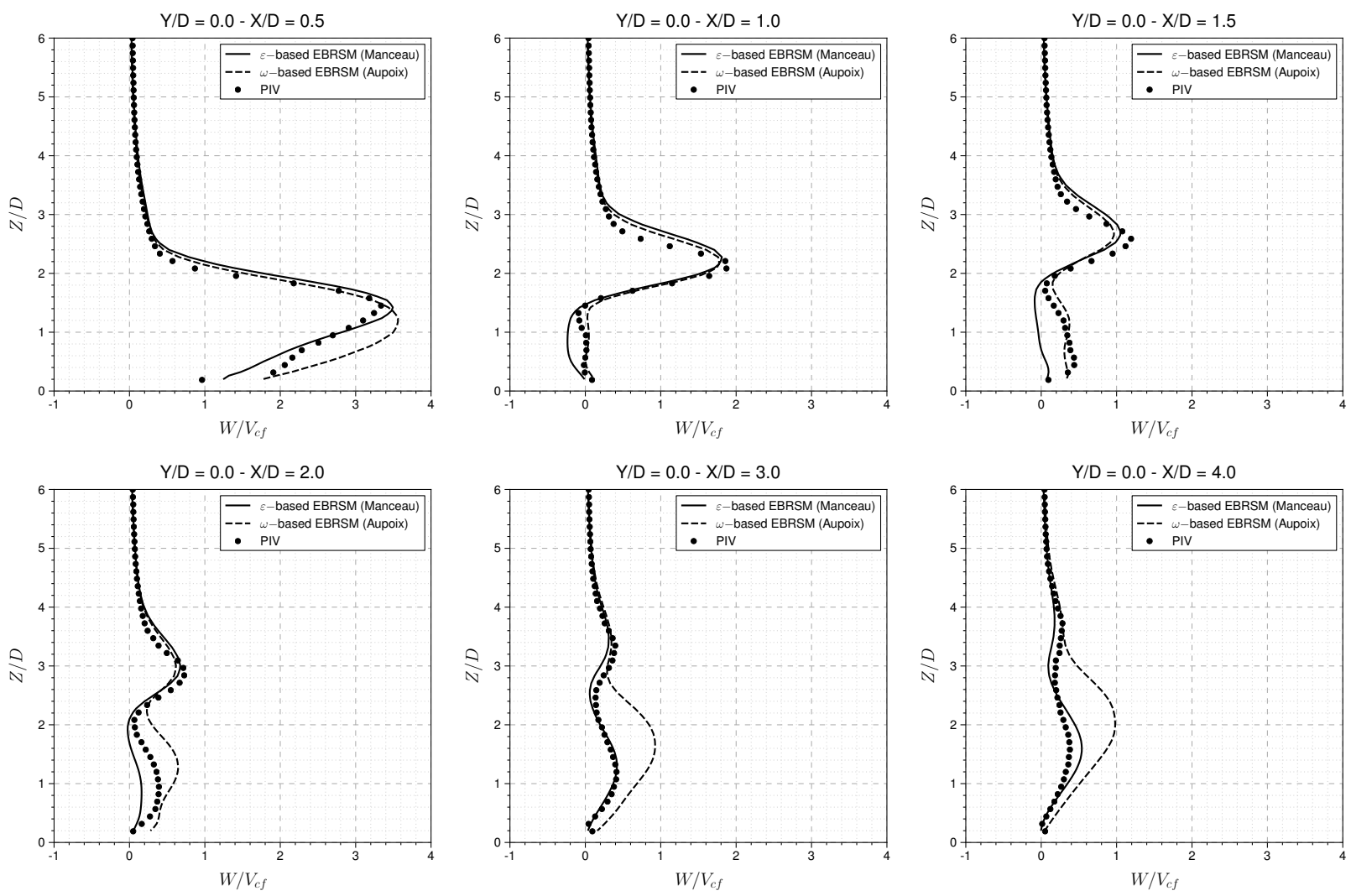

Fig. 6 Profiles of vertical velocity $W$ normalized by crossflow velocity $V_{c f}$ in the centreplane $Y=0$. Simulations performed with EBRSM turbulence models

Fig. 6 presents the profiles of vertical velocity $W$ in the centreplane $Y=0$. One can notice that EBRSM better predict the upper peak location and intensity compared to first order closure turbulence models. At $X / D=0.5, W$ profile is reasonably well predicted by the $\varepsilon$-based model while the $\omega$-based one predicts higher velocity levels near the wall. From $X / D=1$, the upper peak is only very slightly underestimated and shifted upward. The $\omega$-based EBRSM provides a good prediction of $W$ profiles at $X / D=1$ and 1.5. Between $X / D=1.5$ and 2, one can see a rapid growth of the secondary peak. Beyond $X / D=2$, the $\omega$-based EBRSM exhibits a behaviour identical to EARSM or EVM characterised by a significant overestimation of the secondary peak in $W$ profiles. On the contrary, the $\varepsilon$-based EBRSM underestimates the initial growth rate of the secondary peak. As $X / D$ increases, the establishment of the counter-rotating vortices speeds-up and the $\varepsilon$-based EBRSM predictions approaches experimental measurements. As a result, the $\varepsilon$-based EBRSM gives a very good prediction of $W$ profile at $X / D=3$ and very slightly overestimates the secondary peak at $X / D=4$.

Profiles of longitudinal velocity $U$ in the centreplane $Y=0$ are presented on Fig. 77. $U$ profiles prediction is significantly improved with the use of EBRSM turbulence models. One can see that $U$ profile is well predicted at $X / D=0.5$ by both EBRSM. Further downstream, both EBRSM models have the tendency to overpredict $U$ deficit within the wake region. The decay of the velocity overshoot is initially overestimated then slows down as $X / D$ increases.

Jet trajectory and velocity magnitude decay predicted by both EBRSM models are presented on Fig. 8. Up to $X / D=3$ for jet trajectory and $X / D=2$ for $\|\vec{V}\|$ decay, it can be noticed that results given by both models are quite similar. As seen on velocity profiles analysis, they slightly overestimate jet lift-off and $\|\vec{V}\|$ decay. Nevertheless, predictions of $\|\vec{V}\|$ decay given by EBRSM models are less drastic than EVM ones. Further downstream, behaviours of $\varepsilon$-based and $\omega$-based models start to strongly differ as $X / D$ increases. Jet trajectory predicted by the $\omega$-based 

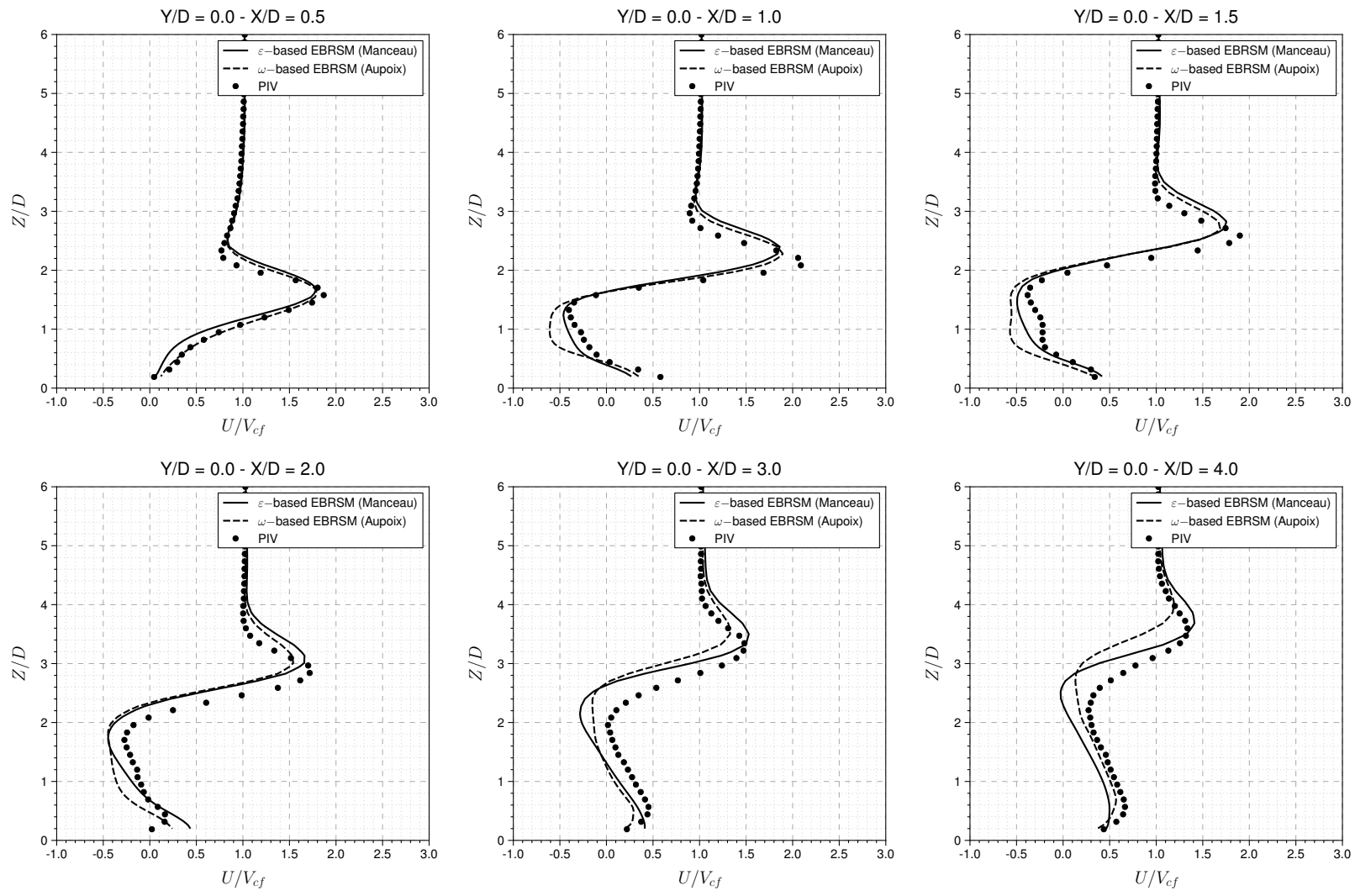

Fig. 7 Profiles of longitudinal velocity $U$ normalized by crossflow velocity $V_{c f}$ in the centreplane $Y=0$. Simulations performed with EBRSM turbulence models

EBRSM follow the same tendency than the one predicted by Hellsten's EARSM. This observation seems coherent with the fact that Hellsten's EARSM is also using a transport equation for $\omega$ to compute turbulence length scale. On the contrary, the $\varepsilon$-based EBRSM perfectly predicts jet trajectory and $\|\vec{V}\|$ decay further downstream. Predicted trajectory is superimposed onto experimental results and $\|\vec{V}\|$ decay curve has the same slope with just a small offset.
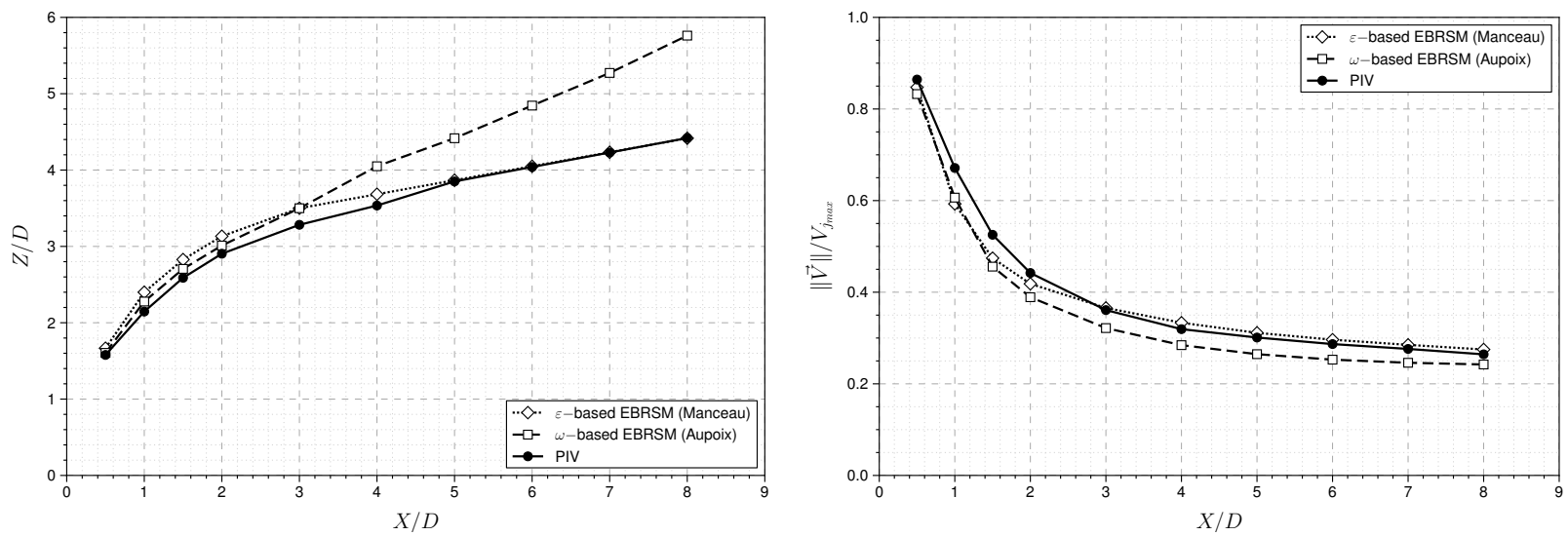

Fig. 8 Jet trajectory based on $U$ maxima in the centreplane (left) and decay of normalized velocity magnitude along jet trajectory (right). 

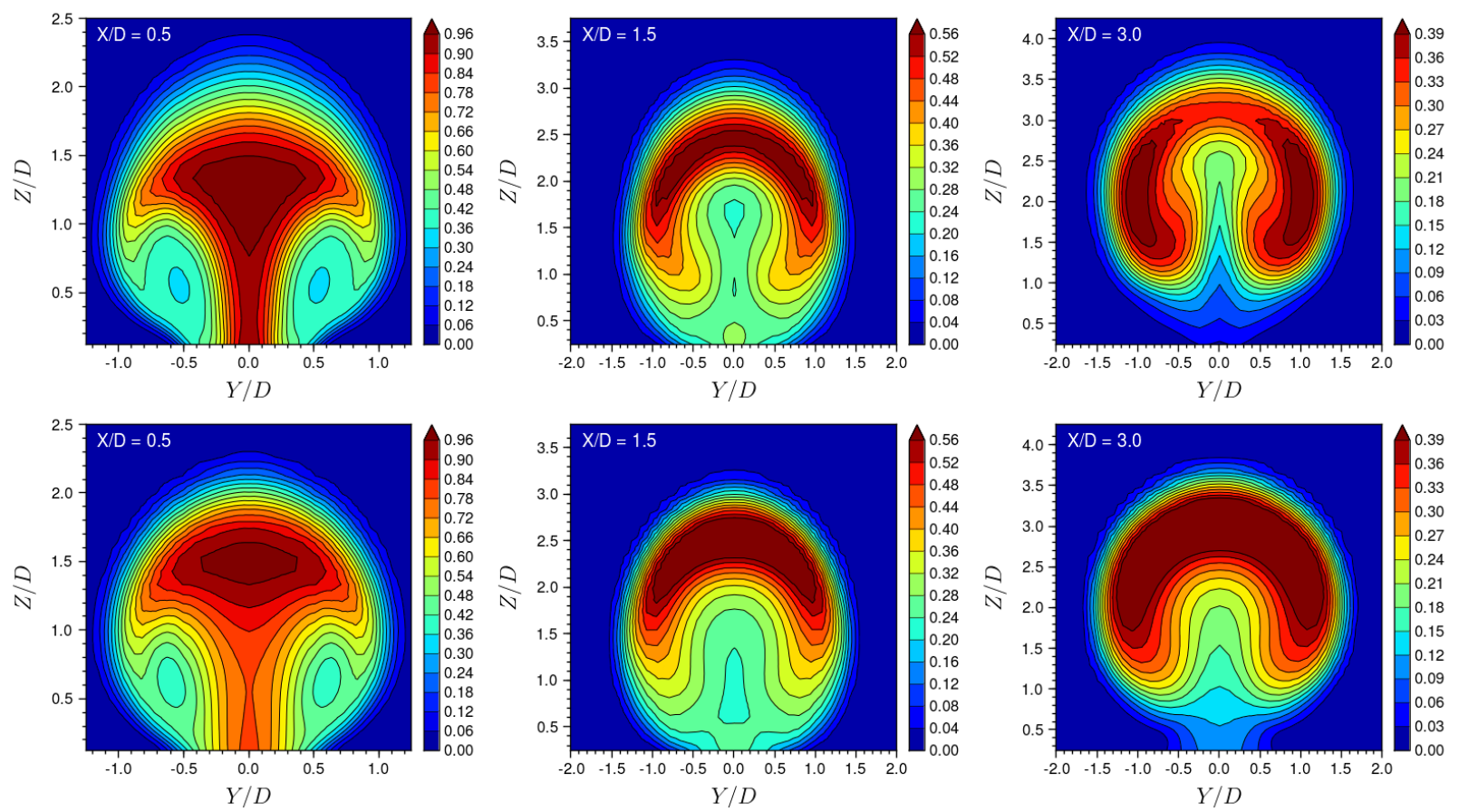

Fig. 9 Normalized temperature fields $\left(T-T_{c f}\right) /\left(T_{j}-T_{c f}\right)$ in three transverse planes downstream jet ejection. $\omega$-based EBRSM [15] (top) and $\varepsilon$-based EBRSM [16] (bottom).

Normalized temperature contours $\left(T-T_{c f}\right) /\left(T_{j}-T_{c f}\right)$ predicted by both EBRSM in three transverse planes located at $X / D=0.5,1.5$ and 3 are presented in Fig. 9 One can see that both EBRSM better capture jet plume diffusion than EVM or even EARSM. This is particularly noticeable in the first transverse plane located at $X / D=0.5$. As introduced before, the $\omega$-based EBRSM model overestimates counter-rotating vortices strength which is detrimental to the correct prediction of the thermal field. Therefore, it exhibits the same drawback as EVM or EARSM turbulence models which is characterised by the rapid migration of temperature maxima towards vortices cores. On the contrary, the $\varepsilon$-based EBRSM model provides a better prediction of the thermal field. In fact, temperature maxima remain located inside jet core which is in better agreement with experimental findings. However, turbulent mixing is still underestimated by the $\varepsilon$-based EBRSM model leading to relatively high temperature levels within jet plume. This underestimation of turbulent mixing is mainly attributed to the fact that the entire turbulent spectrum is modelled in RANS simulations. It is expected that better prediction of turbulent mixing could be achieved with approaches resolving at least part of the turbulent spectrum. However, as the $\varepsilon$-based EBRSM gives fair prediction of the dynamics field, turbulent heat flux modelling may play a critical role in predicting accurate turbulent mixing.

\section{Influence of turbulent heat flux modelling}

Influence of turbulent heat flux modelling has been assessed comparing Gradient Diffusion Hypothesis (GDH) and Daly-Harlow's Generalized Gradient Diffusion Hypothesis (GGDH) [17]. Formulations of both turbulent heat flux models are recalled in equations (11) and (2) respectively.

$$
\begin{gathered}
-\left\langle u_{i}^{\prime} T^{\prime}\right\rangle=\frac{v_{t}}{P r_{t}} \frac{\partial T}{\partial x_{i}} \quad P r_{t}=0.85 \\
-\left\langle u_{i}^{\prime} T^{\prime}\right\rangle=C \frac{k}{\varepsilon}\left\langle u_{i}^{\prime} u_{k}^{\prime}\right\rangle \frac{\partial T}{\partial x_{k}} \quad C=0.3
\end{gathered}
$$

The GGDH model (2) has only been used in combination with EARSM and EBRSM turbulence models as it requires a fair prediction of the Reynolds stress tensor. In the following, results will only be presented for the $\varepsilon$-based EBRSM. However, conclusions drawn for the $\varepsilon$-based EBRSM also hold for calculations performed using the $\omega$-based EBRSM 
or the EARSM coupled to the GGDH model. Normalized temperature contours $\left(T-T_{c f}\right) /\left(T_{j}-T_{c f}\right)$ predicted by the $\varepsilon$-based EBRSM coupled to GDH or GGDH modelling for turbulent heat flux are presented in Fig. 10 Results show that the use of GGDH model for turbulent heat flux enhances turbulent mixing prediction creating an extra diffusion compared to the more classical modelling with a constant turbulent Prandtl number. Therefore, an homogenisation of temperature levels within the jet occurs which goes along with a decrease of temperature maxima. As an example, within the transverse plane located at $X / D=3$, the maximum temperature is $207^{\circ} \mathrm{C}$ with GDH model while it goes down to $183^{\circ} \mathrm{C}$ with the use of the GGDH model. As a reminder, maximum measured temperature in this transverse plane is of $153^{\circ} \mathrm{C}$. It is also worth noticing that results point out that turbulent heat flux modelling only affects temperature levels within jet plume without modifying jet envelope. In fact, the overall jet diffusion within the cross-stream seems unaffected by the turbulence heat flux model.
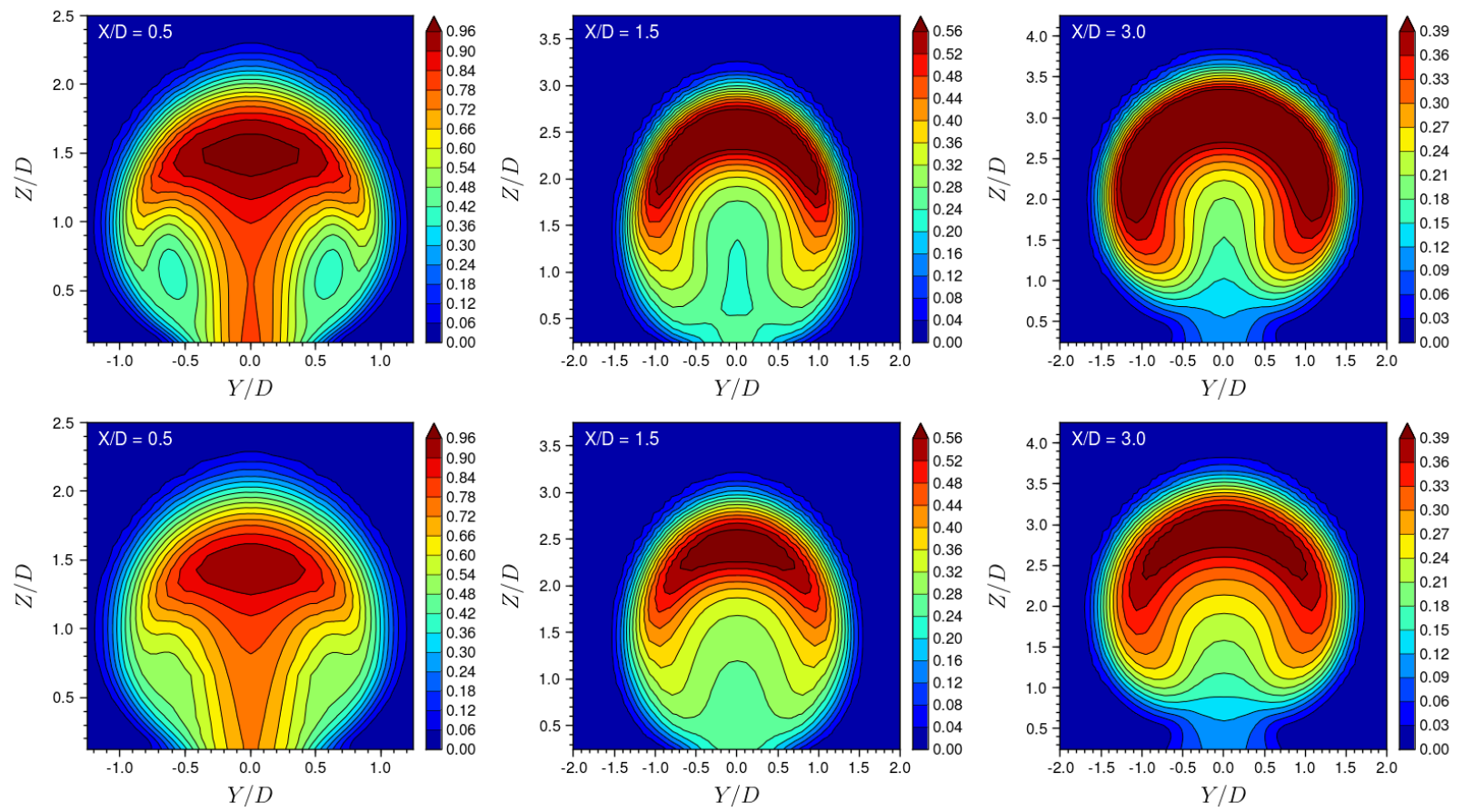

Fig. 10 Normalized temperature fields $\left(T-T_{c f}\right) /\left(T_{j}-T_{c f}\right)$ in several transverse planes downstream jet ejection. $\varepsilon$-based EBRSM [16] with GDH (top) and GGDH (bottom) modelling for turbulent heat flux.

\section{Conclusion}

The present paper investigates the capability of several RANS turbulence models to predict thermal and dynamics fields of a high-temperature jet in a cold crossflow. Calculations performed with turbulence models relying on an isotropic eddy viscosity hypothesis ( $k-\varepsilon$ and $k-\omega \mathrm{SST}$ ) have highlighted their inability to correctly predict counter-rotating vortices intensity as well as turbulent mixing between the jet and the crossflow. The EARSM model proposed by Hellsten [12] which is designed to better capture turbulence anisotropy does not bring major improvement compared to isotropic eddy viscosity models. Nevertheless, it has been noticed that first-order closure turbulence models are qualitatively able to capture main flow features such as the presence of the counter-rotating vortex pair and wake region as well as the initial jet trajectory. Calculations were also performed with higher-order closure turbulence models using two EBRSM models. Results show that the use of such models significantly improves dynamics and thermal fields prediction in the near field. In the far field, differences between the two models increase. On one hand, predictions given by the $\omega$-based model are close to EVM ones and no major improvement is shown. On the other hand, the $\varepsilon$-based model provides fair prediction of dynamics field improving therefore thermal field prediction. From a thermal point of view, results obtained with the EBRSM models are significantly improved when coupled to more complex models for the turbulent heat flux such as the GGDH model. This advanced thermal modelling enhances thermal field prediction creating extra diffusion compared to the GDH model which involves a constant turbulent Prandtl number 


\section{References}

[1] Kamotani, Y., and Greber, I., "Experiments on a turbulent jet in a cross flow," AIAA journal, Vol. 10, No. 11, 1972, pp. 1425-1429.

[2] Harms, L., "Experimental investigation of the flow field of a hot turbulent jet with lateral flow," NASA TT F 15, 707, Vol. Part I, 1974.

[3] Chochua, G., Shyy, W., Thakur, S., Brankovic, A., Lienau, J., Porter, L., and Lischinsky, D., "A computational and experimental investigation of turbulent jet and crossflow interaction," Numerical Heat Transfer: Part A: Applications, Vol. 38, No. 6, 2000, pp. 557-572.

[4] Karvinen, A., and Ahlstedt, H., "Comparison of turbulence models in case of jet in crossflow using commercial CFD code," Engineering Turbulence Modelling and Experiments 6, Elsevier, 2005, pp. 399-408.

[5] Ray, J., Dechant, L., Lefantzi, S., Ling, J., and Arunajatesan, S., "Robust Bayesian Calibration of a $k-\varepsilon$ Model for Compressible Jet-in-Crossflow Simulations,” AIAA Journal, Vol. 56, No. 12, 2018, pp. 4893-4909.

[6] Li, Z., Murugappan, S., Gutmark, E., and Vallet, L., "Numerical simulation and experiments of jets in cross flow," 44th AIAA Aerospace Sciences Meeting and Exhibit, 2006, p. 307.

[7] Bézard, H., Donjat, D., Viguier, P., Jouhaud, J. C., and Bocquet, S., "Projet ASTHER - Analyse et Simulation des jets débouchants et de leur impact THERmique,” Technical Note 2/15118 DMAE, ONERA, December 2012.

[8] Menter, F. R., “Two-equation eddy-viscosity turbulence models for engineering applications,” AIAA journal, Vol. 32, No. 8, 1994, pp. 1598-1605.

[9] Lakehal, D., "Near-wall modeling of turbulent convective heat transport in film cooling of turbine blades with the aid of direct numerical simulation data," Journal of turbomachinery, Vol. 124, No. 3, 2002, pp. 485-498.

[10] Bergeles, G., Gosman, A. D., and Launder, B. E., “The turbulent jet in a cross stream at low injection rates: a three-dimensional numerical treatment," Numerical Heat Transfer, Part B: Fundamentals, Vol. 1, No. 2, 1978, pp. 217-242.

[11] Rusch, D., Blum, L., Moser, A., and Rösgen, T., "Turbulence model validation for fire simulation by CFD and experimental investigation of a hot jet in crossflow," Fire Safety Journal, Vol. 43, No. 6, 2008, pp. 429-441.

[12] Hellsten, A. K., "New advanced k- $\omega$ turbulence model for high-lift aerodynamics," AIAA journal, Vol. 43, No. 9, 2005 , pp. 1857-1869.

[13] Haven, B. A., and Kurosaka, M., "Kidney and anti-kidney vortices in crossflow jets," Journal of Fluid Mechanics, Vol. 352, 1997, pp. 27-64.

[14] Haven, B. A., and Kurosaka, M., "Improved jet coverage through vortex cancellation,” AIAA journal, Vol. 34, No. 11, 1996, pp. 2443-2444.

[15] Aupoix, B., "Improving the coupling of the Speziale, Sarkar and Gatski Differential Reynolds Stress Model with an $\omega$ scale equation," Technical Note 1/13929 DMAE, ONERA, 2012.

[16] Manceau, R., "Recent progress in the development of the Elliptic Blending Reynolds-stress model," International Journal of Heat and Fluid Flow, Vol. 51, 2015, pp. 195-220.

[17] Daly, B. J., and Harlow, F. H., “Transport equations in turbulence,” The Physics of Fluids, Vol. 13, No. 11, 1970, pp. $2634-2649$. 